University of Massachusetts Amherst

ScholarWorks@UMass Amherst

Communication Department Faculty Publication

Series

Communication

2020

\title{
What drives hyper-partisan news sharing: Exploring the role of source, style, and content
}

\author{
Weiai Wayne Xu \\ University of Massachusetts Amherst \\ Yoonmo Sang \\ University of Canberra \\ Christopher Kim \\ University of Canberra
}

Follow this and additional works at: https://scholarworks.umass.edu/communication_faculty_pubs

\section{Recommended Citation}

Xu, Weiai Wayne; Sang, Yoonmo; and Kim, Christopher, "What drives hyper-partisan news sharing: Exploring the role of source, style, and content" (2020). Digital Journalism. 122.

https://doi.org/10.1080/21670811.2020.1761264

This Article is brought to you for free and open access by the Communication at ScholarWorks@UMass Amherst. It has been accepted for inclusion in Communication Department Faculty Publication Series by an authorized administrator of ScholarWorks@UMass Amherst. For more information, please contact scholarworks@library.umass.edu. 
What drives hyper-partisan news sharing: Exploring the role of source, style, and content

\author{
Weiai Wayne $\mathrm{Xu}$ \\ Assistant Professor \\ N334 Integrative Learning Center, \\ Department of Communication \\ University of Massachusetts - Amherst \\ Amherst, MA 01003-1100 \\ Phone: +001-(414)688-3059 \\ Email: weiaixu@umass.edu \\ Yoonmo Sang \\ Assistant Professor \\ Faculty of Arts \& Design \\ University of Canberra \\ Email: yoonmo.sang@ canberra.edu.au
}

Christopher Kim

Ph.D. Candidate

Faculty of Arts and Design, University of Canberra

Email: chouleyoun@gmail.com 
A growing number of hyper-partisan alternative media outlets have sprung up online to challenge mainstream journalism. However, research on news sharing in this particular media environment is lacking. Based on the virality of sixteen partisan outlets' coverage of immigration and using the latest computational linguistic algorithm, the present study probes how hyperpartisan news sharing is related to source transparency, content styles, and moral framing. The study finds that the most shared articles reveal author names, but not necessarily other types of author information. The study uncovers a salient link between moral frames and virality. In particular, audiences are more sensitive to moral frames that emphasize authority/respect, fairness/reciprocity, and harm/care.

Keywords: news sharing, news diffusion, partisan media, Facebook, social media, moral framing, moral foundations theory 


\section{What drives hyper-partisan news sharing: Exploring the role of source, style, and content}

The 2016 U.S. Presidential Election shook the nation, calling us to examine the divisive and deceptive role played by media outlets and internet platforms (Marwick \& Lewis, 2017). It is known that public confidence in media institutions has been declining (Knight Foundation \& Gallup, 2018; Nielsen, Newman, Fletcher, \& Kalogeropoulos, 2019). Mainstream media outlets are increasingly challenged by the partisan alternative journalism represented by Breitbart News and many other ultra-partisan outlets (Benkler, Faris, \& Roberts, 2018). This form of alternative journalism attacks mainstream journalism for its hegemonic and corrupted power in setting the news agenda and narratives (Holt, Ustad Figenschou, \& Frischlich, 2019). It emphasizes its alternativeness by deviating from the mainstream media in styles and narratives, as well as by catering to a largely insulated partisan audience (Benkler et al., 2018).

Hyper-partisan media are blamed as "the principal incubator and disseminator of disinformation" (Faris et al., 2017, p. 19), citing concerns that partisan content tends to elicit more social media engagements than mainstream media content (Silverman, 2016). While hyperpartisan, alternative journalism is known to benefit from social media platform architecture and algorithms to maximize virality, our knowledge is still limited concerning factors related to partisan news sharing in the social media environment (Tucker et al., 2018). Built on existing literature on general news sharing behavior (see Valenzuela, Piña, \& Ramírez, 2017 for review), this study pays specific attention to partisan news sharing behavior. Guided by Aristole's notion of ethos, logos, and pathos, and drawing from the literature on media trust, dual information processing, moral foundations, and news values to focus on three factors, namely, source transparency, content styles, and moral framing. These factors were shown to significantly predict news "shareworthiness" for mainstream media (dos Santos, Lycarião, \& de Aquino, 
2019; Kim, 2015; Kümpel et al., 2015; Trilling, Tolochko, \& Burscher, 2017). However, it is not clear how they relate to news "shareworthiness" in a hyper-partisan media environment. In what follows, we situate the factors in the hyper-partisan content, considering various ways partisan audiences perceive media trust and selectively process information, as well as how hyperpartisan alternative journalism departs from traditional journalistic values and norms.

\section{The rise of hyper-partisan alternative media outlets}

Hyper-partisan alternative media outlets are on the rise (Holt et al., 2019), particularly on the political right (Marwick \& Lewis, 2017). The alternative outlets brand their "alternativeness" through opposition to the alleged hegemonic, corrupted, and elitist mainstream media (Holt et al., 2019). In doing so, the alternative outlets position themselves as "outsiders" of the system who give voices to marginalized people and under-covered stories (Figenschou, \& Ihlebæk, 2019) and invite citizen and anonymous reporting (Holt et al., 2019). The early form of alternative journalism was grassroots-oriented and positioned mostly in the progressive left, but has been recently appropriated by the alternative right to brand their own counter-hegemonic and anti-elite media agenda (Holt et al., 2019). Exemplifying this new form of alternative journalism is Breitbart News, which has become the spokesperson for the ethnonationalist movement called the Alt-Right (Benkler et al., 2018; Marwick \& Lewis, 2017). The boundary between professional journalism and alternative journalism has also been blurred (Figenschou \& Ihlebæk, 2019). It becomes debatable as to whether content propagated by the hyper-partisan outlets can be regarded as news at all. This is because the alternative media ecosystem consists of not just partisan blogs and news outlets but also fake news and clickbaity sites (Tandoc, Lim, \& Ling, 2018). Holt et al. (2019) called for more research concerning the reach of hyper-partisan alternative media, audience motivation and gratification. This study explores news sharing 
behavior as a way to gauge the influence of the alternative media and to understand the preference, and motivation of their partisan audiences.

\section{News sharing}

News sharing facilitated by social media has redefined the reach and effect of news; unlike faceto-face interactions, where the reach and effect of news sharing are limited to a person's immediate social circles, news sharing on social media can impact a large and undefined audience due to social media's network effect and personalization algorithms (Trilling et al., 2017). Several factors make social media-based news sharing conducive to the echo chamber effect (Wang et al., 2017), causing the further spread of disinformation and partisan bias (Benkler et al., 2018). First, the consumption of media content is increasingly driven not by seeking the most informative content but by the validation of existing beliefs (Fisher, 2016). Second, people are somewhat reluctant to share content that contradicts the prevailing values and beliefs in their social circle (Liu, Rui, \& Cui, 2017); they may be inclined to share news that conforms to the expectation of a social group or share it only with like-minded people (Wang, Hmielowski, Hutchens, \& Beam, 2017). Such socially motivated news sharing decisions can be facilitated by inferring from a wide range of bandwagon heuristics inductive of content popularity within a social circle, such as the number of likes and shares; audiences use such heuristics to judge the trustworthiness and relevancy of the content, resulting in a self-fulfilling cycle (Tandoc et al., 2018).

An extensive body of literature deals with what factors predict news sharing (dos Santos, Lycarião, \& de Aquino, 2019; Kim, 2015; Kümpel et al., 2015; Trilling et al., 2017). Some research has investigated audience factors through the theoretical lens of uses and gratifications and user characteristics (Kalogeropoulos et al., 2017; Lee \& Ma, 2012). Such studies typically 
use the survey/experimental design for reaching causal conclusions. Nevertheless, the ecological validity of the studies is constrained (Trilling et al., 2017). To overcome the shortcoming, another body of studies relies on observed behavioral data to examine content-level factors (García-Perdomo et al., 2018; Trilling et al., 2017). It draws on a number of theoretical frameworks to discover factors of content "shareworthiness," including news values, which posits what types of stories are favored by journalists and news audience alike (Eilders, 2006; Trilling et al., 2017); news framing, which deals with how selective presentations of issues and events invoke different audience responses (Valenzuela et al., 2017); information processing, arguing that certain heuristic cues are intrinsically more attention-grabbing (Chaiken, Liberman, \& Eagly, 1989), which consequently relates to more sharing; and lastly, audience expectations of journalistic transparency and interactivity amidst waves of digitalization of news production (Fletcher \& Park, 2017; Jahng \& Littau, 2016).

Through synthesizing the theoretical lens used in previous works, we narrowed the focus to three content and source-level factors to be discussed in-depth (i.e., source transparency, content styles, and moral frames). The three factors map onto the notion of Aristole's ethos, logos, and pathos. To the best of our knowledge, the current study is one of the earliest attempts to investigate these factors as predictors of hyper-partisan news sharing. This study does not assume hyper-partisan news sharing to be fundamentally different from conventional news sharing. It does, however, recognize the uniqueness of hyper-partisan outlets in their predominantly sensational story-telling, the moralization of public issues, and in their partisan audience base who may have a different perception of media trust than the general news audience.

\section{Ethos: Source transparency for media trust}


Ethos relates to convincing the audience through the source's credibility and character. To that end, media trust is essential to audience engagement with news content (Fletcher \& Park, 2017; Jahng \& Littau, 2016). Trust in traditional journalism rests upon objective, balanced, and expert-based reporting (Lee, 2015). However, trust in the hyper-partisan alternative media outlets is likely ideologically entangled. Put differently, media trust and perceived journalistic quality hinge in part upon whether content creators' ideological stance aligns with that of the audience (Metzger et al., 2015; Meyer et al., 2010). Conservatives along with populists in the US have labeled mainstream media "fake news" and decry "liberal bias" (Lischka, 2019), whereas the same "fake news" phenomenon is extensively tied to the manipulated and misleading pro-Trump coverage during the 2016 election (Bakir \& McStay, 2018). Also challenging the notion of media trust is the digitalization of the newsroom as content creators join social media. Content creators need to adapt to a digital environment that values social presence, interactivity, and transparency (Jahng \& Littau, 2016; Lee, 2015). As shown in Jahng and Littau's (2016) study, journalists who are active on social media are perceived as more credible. In a nutshell, media trust in the digital age and partisan era is based upon ideological alliance and interactivity, to which transparency is the key.

Transparency is about making the editorial production process open to the public so that the audience can directly interact with media outlets to provide feedback and to learn about media outlets' ideological stances. Transparency is specifically manifested in revealing information about authors and using external sources to support media outlets' presentation and interpretation of current affairs (Chadha \& Koliska, 2015). An experiment by Curry and Stroud (2017) found that a news article is perceived as more credible when it reveals the author's name, 
picture, and affiliation. Revealing such author information shows a willingness to invite public scrutiny of a media outlet.

Media content also typically presents the authors' social media presence, which is a sign of interactivity (Jahng \& Littau, 2016). Additionally, author information provides social cues that can reduce the social distance between content creators and the audience. According to the social presence theory, people become attentive to the scarce social cues to form impressions when they communicate in a virtual environment where verbal cues are lacking (Walther, 1993).

Transparency is relevant to hyper-partisan alternative media outlets for two competing reasons. Disclosing author information allows the audiences to study and vet the authors' ideological stances. The information even helps like-minded audiences develop a para-social relationship with partisan content creators — such relationship is linked to stronger perceived source credibility (Landreville \& Niles, 2019). Partisan media figures (such as Rush Limbaugh, Andrew Breitbart, and Alex Jones) and their personal brands play a pivotal role in defining contemporary partisan politics (Jutel, 2018). Hyper-partisan outlets may even use the disclosure to create a façade of accountability to legitimize their dubious content. Meanwhile, some hyperpartisan outlets may emphasize on anonymous contributions to be distinguished from traditional journalism (Holt., 2019). In short, while traditional journalism calls for transparency, the incentives for hyper-partisan outlets to adopt the transparency principle vary. We approach the role of transparency through an exploratory research question.

RQ1: What is the relationship between the amount of author details disclosed in news articles and news sharing? 
Another way to build up public trust is by citing source materials and evidence through hyperlinks (Coddington, 2014; Chadha \& Koliska, 2015). The hyperlinking practice serves as "a journalistic parallel to the academic reference system” (Mor \& Reich, 2018, p. 1092). Early studies found that alternative outlets (e.g., political bloggers and citizen journalists) had extensively used hyperlinking to cite raw materials and data, whereas their mainstream counterparts preferred internal links to formal publications (Mor \& Reich, 2018). By citing sources through hyperlinks, media outlets provide readily available background information to audiences, allowing the audiences to verify claims and the authenticity of content themselves. We expect that source-citing to be an audience-friendly practice that provides richer information and communicates a commitment to quality reporting. Hence, H1 is proposed.

H1: The amount of external sources cited via hyperlinks in news articles positively predicts news sharing.

\section{Logos and pathos: Content styles for attention}

Traditional journalism is thought of as objective and 'just the facts.' The rational style and its deemphasis on emotion have long been a marker of distinction between professional journalism and tabloid journalism (Peters, 2011). Emotion is often, albeit unfairly, dismissed, and conflated with sensational coverage (Peters, 2011; Wahl-Jorgensen, 2019). The norm of rationality in news

production, that is presenting logical, critical, and evidence-based narratives are rooted in Aristotle's logos (appealing to reason). An audience hence forms impressions partly based on the structure and style of online content (Choi \& Stvilia, 2015; Rowley \& Johnson, 2013).

In relations to Aristotle's pathos (appealing to emotions), recently, a growing number of studies have paid increased attention to emotion's "role in the production, texts and audience 
engagement with journalism” (Wahl-Jorgensen, 2019, p. 5, emphasis in original; Zou, 2018).

Compared to traditional journalism that values rationality and objectivity, the contemporary hyper-partisan alternative journalism is sensational, featuring dramatic events and appealing to audiences' emotional responses with intensified or dramatic language (Mourão \& Robertson, 2019). Online space is filled with emotions as collective grievance attracts like-minded people and affective storytelling drives affective publics to take actions (Papacharissi, 2015). The use of emotion in reporting corresponds to Aristotle's pathos (appealing to the emotions) and is supported by ample evidence concerning the persuasiveness of emotional appeals (Bail, Brown, \& Mann, 2017; Kilgo, Lough, \& Riedl, 2017; Stieglitz \& Dang-Xuan, 2012; Veltri \& Atanasova, 2015).

It is also a salient factor of news sharing (Berger, 2011; Khuntia, Sun, \& Yim, 2016; Kim, 2015). Several theories explain why the use of emotion is prevalent and effective in information diffusion. First, emotion is one of the core news values (Trilling et al., 2017). Recall that news values are a set of characteristics that make certain news stories more appealing to journalists and audiences (García-Perdomo et al., 2018; Trilling et al., 2017). Second, sharing emotional news is a natural process of creating social bonding and adapting to traumatic events (Harber \& Cohen, 2005). Noteworthy is that emotional contagion online even occurs without the audience's awareness: Kramer and colleagues (2014) observed that when Facebook users were exposed to negative content in the Facebook timeline, they subsequently expressed more negative thoughts in posts. Third, in terms of information processing, people are wired to be more attentive to emotional cues, making emotional language and content an effective tool in eliciting attention and attitude change (Chaiken, Liberman, \& Eagly, 1989).

H2a: The strength of emotion presented in news articles positively predicts news sharing. 
RQ2: What is the relationship between the expressed rationality in news articles and news sharing?

On top of the rational versus emotional style difference that differentiates traditional and alternative journalism, another style factor studied in the literature is the use of audiovisual content (Tucker et al., 2018). Newsrooms use multimedia journalism to arouse the audience's engagement (Pincus, Wojcieszak, \& Boomgarden, 2017). Multimedia content provides "realism cues" to create a more direct sensory experience (Sundar, 2008) and is effective in stimulating audiences (Tucker et al., 2018). A wealth of evidence suggests a positive link between including multimedia content and a higher chance of information sharing (Chung, 2017; Liu et al., 2017), including the sharing of partisan content (Tucker et al., 2018).

H2b: The use of multimedia content in news articles positively predicts news sharing.

\section{Pathos: Moral framing}

Framing is one of the most discussed journalistic practices (Entman, 1993; Ryan, Carragee, \& Meinhofer, 2001). Recently, a growing number of studies have explored framing as a determining factor in news sharing (Valenzuela et al., 2017). To frame a story is to selectively present and emphasize certain aspects of events by using specific keywords, phrases, sources, and examples (de Vreese, 2012). Framing produces certain interpretations of events that are deemed desirable by the news audience and the newsroom. Hence, framing is frequently used by ideologically leaning news outlets to promote certain partisan narratives (Lowry, 2008). Commonly used frames are economic consequences, conflicts, human interest, and morality (Valenzuela et al., 2017). 
The current study focuses on a particular type of framing called moral framing. Moral framing refers to the practice of putting "the event or issue in the context of values, moral prescriptions, normative messages, and religious or cultural tenets" (Semetko \& Valkenburg, 2000; Valenzuela et al., 2017, p. 809). The focus on moral frames is based on two reasons. First, past research shows that moral framing is a particularly salient predictor of news sharing. Specifically, in a comprehensive examination of how different news frames (e.g., economic consequence, conflicts, morality, etc.) drive sharing, Valenzuela and team (2017) found that moral frames are associated with increased news sharing, whereas the other frames decrease sharing. Although moral frames are not as frequently used as other frames, they are the primary perspective through which the audience understands current events (Valenzuela et al., 2017). Valenzuela and colleagues (2017) argued that moral framing drives sharing because moral frames tend to invoke strong emotions. Moreover, moral frames convey social values that the audience likely identifies with - an important factor to consider in the partisan age and amidst cultural wars (Brady et al., 2017). The second reason is that moral frames correspond to different moral foundations, which can be seen as the root causes of policy disagreements and partisan divides (Graham, Haidt, \& Nosek, 2009). It needs to be recognized that moralizing public issues to engage audiences is not unique to hyper-partisan journalism: in the book New Class War, Lind (2019) criticized the mainstream media's moral framing of populist and culturally conservative voters as brainwashed and intolerant. However, considering the political agenda of hyperpartisan journalism, we assume that moral framing is particularly salient in hyper-partisan media's approach to content.

While the existing literature on news sharing shows a positive link between moral frames and news sharing, no current study explores how specific moral frames are associated with 
sharing. Moral frames can be constructed through different moral foundations (Haidt \& Graham, 2007). The moral foundations theory posits that people of different ideological camps rely on different moral intuitions to justify actions and attitudes (Graham, Haidt, \& Nosek, 2009). The differences in moral foundations can account for the root causes of partisan divides in the socalled culture war in America (Graham et al., 2009). Five moral dimensions are proposed by the theory: Harm/care, fairness/reciprocity, ingroup/loyalty, authority/respect, and purity/sanctity.

Harm/care and fairness/reciprocity are linked to the ethics of care and justice (Graham et al., 2009). The two moral foundations involve protecting, caring, and nurturing the vulnerable, with an emphasis on the value of individual human beings - a social value widely embraced by individualistic western cultures. Nevertheless, many non-western cultures embrace moral foundations that value groups over individuals, hence forming the other three moral foundations: ingroup/loyalty, authority/respect, and purity/sanctity (Graham et al., 2009). The three foundations stress duty, commitment, and self-control, rather than individual autonomy (Graham et al., 2009). Studies show that the first two dimensions, namely harm/care, fairness/reciprocity, are the dominant moral foundations embraced by liberals, whereas the conservatives endorse and use the five foundations more equally (Graham et al., 2009).

As hyper-partisan outlets often cover socially and morally divisive issues (e.g., abortion, immigration, healthcare, etc.) and such issues become the major vehicles for conveying partisan agendas, we expect a salience of moral framing in partisan news sharing:

H3: News sharing is related to the presence of moral frames.

RQ3: What is the relationship between news sharing and different moral frames based on moral foundations? 


\section{Methods}

Sample choice and data cleaning

Facebook was chosen as the site of investigation, given that it is one of the most widely used social sharing platforms in the U.S. (Perrin \& Anderson, 2019). To identify hyper-partisan outlets on Facebook, we first used Benkler et al. (2017) and Tucker and colleagues' (2018) work to create a preliminary list of 52 hyper-partisan sites. Based on the Alexa web traffic data, we then selected the ten most popular and ten least popular sites from the list to ensure the representativeness of media outlets of varying degrees of influence. We identified the outlets' Facebook pages (one outlet was dropped from the list due to its absence on Facebook). Table 1 shows a list of outlets selected and their Facebook activities. The top outlets are predominantly conservative, while the liberal outlets tend to be among the least popular in terms of web traffic. Between 11/08/2015 and 11/08/2016, the top outlets produced a total of 83,181 Facebook posts, and the bottom-ranked outlets produced 44,398 posts.

The final list had 17 outlets, including the conservative Breitbart, InfoWars, The Daily Caller, Conservative Tribune, and the liberal outlets Addicting Info and Truthout. To control for different news outlets' topical preference, the study used the single case design, focusing on the coverage of immigration. The issue of immigration galvanized robust media attention during the 2016 U.S. presidential election, fueled by then-candidate Donald Trump's calls for building the border wall and a travel ban targeted on certain Muslim nations, as well as his strong stance on illegal immigration and the deferred action for childhood arrivals (DACA). We used a customized Python script to collect the selected news outlets' public Facebook posts. At the time of the study, Facebook had a public API to allow researchers to download any public page's posts. However, the public API has become restricted after the Cambridge Analytica scandal. 
We identified immigration-related articles based on a set of search criteria: the articles must be the original content produced by the selected outlets and shared by the outlets as link posts (a type of Facebook posts in which users insert a URL and add commentary); Facebook post content, and page descriptions should contain at least one of the following keywords: immigration, immigrants, immigrant, travel ban, DACA, dreamer, and illegal alien. Table 1 breaks down the outlets by the total number of their Facebook posts and the number of posts about immigration. In total, there were 1,805 link posts before the data cleaning.

We then used Newspaper3k, a Python package for article scraping, to extract article content from links in the Facebook posts. Aidded by a manual valiation, the process identified 128 posts with expired URLs which indicate deleted content; . In the remainder of 1,677 posts with unexpired website content, we further removed 302 posts linking to webpage content containing predominantly videos/photos rather than text. We recognized that removing the predominantly multi-media content may limit the generalizability of our findings. However, it is a necessary compromise due to the use of text-based measures for linguistic characteristics. In the cases where duplicated content is found, the likes and shares of articles were averaged to control for media outlets' cross-posting. The final sample included 1,156 unique immigrationrelated articles.

INSERT TABLE 1

Measures

To measure the independent variables in the study, we used both manual coding and computational techniques. To ensure the accuracy of the manual coding, the coding task was 
equally divided among three coders. The third coder checked the entire coding results to correct any discrepancies.

Source transparency. Recall that source transparency is operationalized as (1) revealing author's details and (2) citing through external hyperlinks. We manually inspected each article, checking whether an article revealed the author's name, photo, bio, and email address/social media handles. We coded the presence of the above information on the article page or on a separate webpage linked to the article. The numbers: $84 \%$ of the articles disclosed the author's name (articles without author name typically use the outline name as the byline); $47 \%$ of the articles contained author photos; $51 \%$ had information about an author's contact and social media handles, and 54\% showed the author's bio. We then counted unique external sources cited via hyperlinks per article. With the focus on external sources, any internal hyperlink (hyperlinking to content produced by the same outlet) was excluded. To count the hyperlink sources, we used a Python package to extract all hyperlinks present on an article page and aggregated the external links based on the same domain name (i.e., separate links to different New York Times articles will be counted as one because they all come from the same web source). We then removed links to social media and common web platforms (e.g., Twitter, Instagram, Reddit, Facebook, YouTube, Tumblr, Apple, Amazon, and Google); we also removed links to news aggregation sites (e.g., feedburner) and links embedded for audience tracking (e.g., quantacas and breezy). On average, an article contained more than three hyperlinked sources.

Content styles were unpacked into three variables: (1) the strength of emotion, (2) the strength of expressed rationality, and (3) the amount of multimedia content. The first two variables for content styles were measured using the dictionary-based linguistic analytic program LIWC (Linguistic Inquiry and Word Count) (Pennebaker, Boyd, Jordan, \& Blackburn, 2015). 
LIWC scans texts and calculates the prominence of about 90 types of words and symbols (Pennebaker et al., 2015). First, the strength of emotion was measured by the affect score in LIWC. The Affect score calculates the proportion of emotion words in text with a higher score indicating a stronger emotion $($ mean $=4.10, \mathrm{sd}=1.72)$. Second, the strength of expressed rationality was gauged by Analytic score in LIWC (on a 0-100 scale). The use of Analytic words (mean $=84.84, \mathrm{sd}=15.06)$ indicates a logic and sophisticated thinking, as opposed to intuitive and narrative thinking (Pennebaker et al., 2015). Lastly, the amount of multimedia content was measured by counting story-related pictures/videos on the article page $($ mean $=1.39, \mathrm{sd}=1.45)$ (not including advertising content and multimedia elements in the recommended/related article section).

Moral frames were measured by applying the moral foundations dictionary (MFD hereafter) (Graham et al., 2009). The dictionary includes 295 words for each of the five foundations, namely, harm/care, fairness/reciprocity, group/loyalty, authority/respect, and purity/sanctity (Graham et al., 2009). The words for each moral foundation cover a bi-polar continuum between foundation-supporting (referred to as virtues) and foundation-violating (referred to as vices). Following previous studies using MDF (Garten et al., 2016; Hoover et al., 2018), we used the Distributed Dictionary Representations (DDR) approach to apply MFD (Garten et al., 2018). DDR is different from the dictionary-based word count approach (such as the one used in LIWC). Rather than counting the appearance of dictionary words, DDR accounts for semantic relations of the terms in the dictionary (Garten et al., 2018) Hence, DDR is advantageous (see Garten et al., 2018): it allows researchers to count not just the appearance of dictionary words but words contextually and semantically similar to the dictionary words, which can address dynamic and generative aspects of language use. To implement DDR, we used the 
open-source Python code provided by Garten and colleagues (2018). The codes first generate a Word2Vec model using the pre-trained Google News Corpus containing (3 billion running words) word vector model (3 million 300-dimension English word vectors)and then compute the similarity score between dictionary words and the studied text.

The dependent variables were the number of shares and likes of a Facebook post linking to a selected article. The two variables reflected two levels of news sharing. Liking an article does not have the same level of significance as sharing does. But liking an article may drive future sharing because it makes the article more visible to a user's friends. The shares and like count were obtained from the Facebook API, capturing the scope of news diffusion at the moment of data collection. If the same article was shared multiple times, their likes and shares were averaged. On average, an article in the study dataset was shared 862 times $(\mathrm{sd}=1,785)$ and liked 1,526 times $(\mathrm{sd}=3,165)$.

The model had the following control variables: (1) the selected news outlets' Facebook page follower counts. On average, a selected outlet had a follower count of 2.58 million ( $\mathrm{sd}=$ 1.83 million); (2) word count of an article (mean $=532 ; \mathrm{sd}=489) ;(3)$ the length of time since posting, under the assumption that earlier posts tended to receive more shares than the more recent ones. The length of time since posting was calculated as the day difference between the last day of data collection (04/19/2016 for Breitbart and 06/12/2017 for the rest) and the day an article was posted. On average, an article had been on Facebook for a year at the time of data collection. Table 2 shows the descriptive statistics for all variables in the model. 


\section{Results}

We started by fitting the dependent variables to different distributions (e.g., normal, Poisson, negative binomial, and log-normal). Similar to dos Santos, Lycarião and de Aquino's (2019), we found that the dependent variables were close to a log-normal distribution: The skewness of share count was 5.77, while its logarithm's skewness was -0.33 . The skewness of like count was 6.67, while its logarithm's skewness was -0.31 . Based on this, our analyses use the logarithm of each of the dependent variables.

The unit of analysis is each article content. Because styles and formats of the articles are likely dependent on each news outlet's practices, hence the measures of the article-level source transparency, content styles, moral frames are not independent of each other. To address this issue, we applied multilevel regression analyses using the lme4 package in R. The Facebook page IDs of the selected news outlets were used as the random effect variable. Each model was checked for multicollinearity (using VIF scores), homogeneity, and independence, as well as normality of residuals. We found a strong positive correlation between the five virtue-related and five vices-related moral frames variables. That means if an article emphasizes the violation of a moral foundation, it also likely stresses the support of such a moral foundation. Consequently, the initial model including all of the moral frames had VIF scores between 8 and 14 for most of the moral frame variables. To prevent the multicollinearity issues, the final model includes only moral virtue variables, in which the VIF scores range between 1 and 4 , indicating that the multicollinearity issue is largely tolerable. Table 3 shows the two multilevel regression models with shares count and likes count as the dependent variables respectively. For an easier comparison in explanatory power, the variables were rescaled to have a mean of 0 and a standard deviation of 1 . 
We summarize findings by the order of hypotheses and research questions. Concerning source transparency (RQ1), revealing author name consistently predicted more sharing and liking across the four models; However, articles showing author bio were significantly less shared and liked. $\mathrm{H} 1$ posited that the number of external sources cited via hyperlinks positively predict news sharing. This hypothesis was not supported. Articles with more external hyperlinked sources tended to receive fewer likes.

Concerning content styles, $\mathrm{H} 2$ a predicted a positive relationship between the strength of emotion in articles and news sharing. The support of the hypothesis was mixed. More emotional content was only significantly liked more but it had no significant impact on actual sharing behavior. Regarding the relationship between the expressed rationality and news sharing (RQ2), we found no significant relationship between a formal and logical writing style and like and share counts. $\mathrm{H} 2 \mathrm{~b}$, which predicted a positive association between the use of multimedia content and news sharing, was not supported due to the non-significant role of the number of videos and images in articles.

Lastly, H3 was supported as multiple moral frames were significantly associated with news sharing. Specifically, articles that presented a moral frame emphasizing the support of authority/respect were significantly shared and liked more, whereas articles emphasizing the support of the fairness/reciprocity value were significantly less shared. Additionally, articles mentioning the support of the care moral value received significantly more share, yet fewer likes.

\section{Discussion}


We conducted this study to fill two gaps in the current literature on news sharing behavior. The first gap is a lack of systematic examination of content and source characteristics associated with news sharing in the hyper-partisan media environment; the second gap is a lack of attention paid to content framing through moral lenses. The study uses a mixed-methods design relying on both manual content analysis and computational linguistic analytics, which includes the last Distributed Dictionary Representation algorithms. Although the study is limited by its crosssectional design, findings do reveal a potentially powerful role of moral framing as well as source transparency. We believe our preliminary and correlational findings can pave the ground for future causal testing.

Our argument concerning source transparency is based on the premise that media outlets need to be held accountable by being transparent about who writes what. Source transparency is a journalistic norm upheld in traditional rooms (Chadha \& Koliska, 2015) and is associated with media trust and positive audience engagement (Curry \& Stroud, 2017). We also maintain that the partisan audience may expect different types of source transparency due to their partisan leaning and selective information processing. Study findings show that being transparent remains an important factor, but perhaps what matters more is what kind of information is revealed to the public: At the minimum, it appears that outlets need to reveal authors' names. Holt and colleague (2019, p. 4) observed that alternative media have been more welcoming to "a democratic bottomup inclusion of non-professionals," allowing non-professionals to anonymously publish their own content. Nevertheless, the vast majority of the studied outlets reveal authors' names and the partisan audience does not seem to prefer anonymous submissions. It could be that anonymously produced content is generally of lower quality, thus lacking appeal to the audience. It is also plausible that audiences use the disclosure of author name as a heuristic cue for media trust or 
content legitimacy (Sundar, 2008). Amid the concern over how anonymity affords the spread of disinformation and partisan propaganda (Marwick \& Lewis, 2017), this finding could be read as a reassuring sign that the most viral content, regardless of its partisan biases, has human faces behind it. What was unexpected though was that revealing too much author information was linked to less sharing - this is contrary to what social presence theory predicts and what the norm of interactivity demands (Jahng \& Littau, 2016, Lee, 2015). It is worth noting that in Jahng and Littau's (2016) study, which explored journalists' online social presence, the study participants were specifically asked to rate the credibility of journalists. In the current study, it is not clear whether the audience considers the authors' credibility before they share the news. We suspect that the audience pays limited attention to who writes the article, as they are constrained by their fragmented attention. The audience might be more likely drawn to other attention-grabbing elements such as sensational content and clickbaity titles. Another possibility is that partisan audiences, who look for opinion congruence (Metzger et al., 2010; Meyer et al., 2010), use media outlets as the heuristic for ideological leaning. Since the studied partisan outlets already have clearly defined editorial slants, the audience may not necessarily use the author cues for inferring about a particular piece of content's editorial posture. Alternatively, there is the possibility that the audience is already familiar with the authors, thus just knowing their names provides enough information to form a judgment. In synthesis, the finding suggests that the norm of transparency, which is widely adopted by the mainstream journalism, has relevance for hyperpartisan alternative journalism but the extent to which it may affect content popularity is a function of not how much information but what kind of information is shared with the audience.

Our argument regarding content styles is first grounded in the debate on rational appeals versus emotional appeals. We find a quite limited role of content styles in news sharing - content 
styles only matter so much as to the liking of the content (as opposed to the actual sharing). This is somewhat surprising given prior studies that reveal a salient role of emotion and multimedia content in virality (Kilgo, Lough, \& Riedl, 2017). Hyper-partisan journalism is known for its sensational coverage (Mourão \& Robertson, 2019), the finding casts doubt over the impact of such sensational approach to news. It may invoke positive audience engagement (in the form of liking) but may not result in a wider dissemination of the content. A plausible explanation is that media sensationalize news events not just through using emotional words but through the use of moral frames (the factor to be discussed next). Moral frames generally invoke strong emotions (Valenzuela et al., 2017). The negative link between using multimedia and liking warrant further investigation. One reason for that is having too many multimedia cues causes cognitive overload and affects user experience.

The most significant aspect of our findings is about the salient role of moral framing in driving virality. This link is well-supported by previous studies that identify a link between framing and news sharing (Valenzuela et al., 2017) and the latest moral foundations theory on how partisan divides can be traced back to different moral intuitions on which people form opinions (Graham et al., 2009). What is particularly interesting is our discovery of the divergent effects of different moral frames. First, the audience appears to be more sensitive to content using the authority/respect frame. Note that this moral frame is indicated by words related to law, obey, honor, respect, and such. A post-hoc analysis reveals that the articles most representative of this moral frame are those discussing the constitutionality of immigration policies and proposals under President Obama, the enforcement of border protection. In short, this moral frame is centered around the law and enforcement aspect of the immigration issue. The finding seems to confirm the existing research that ties support for Trump supporters' conservative 
values over social groups, order, and authority (Choma \& Hanoch, 2017). Second, the use of the moral frame of fairness could deter sharing. This moral frame is represented by using keywords similar to rights, justice, fairness, and equity. The topics in the top articles representing this moral frame are mixed, ranging from criticism of Trump's violation of minorities' rights to an endorsement of Trump's proposed ideological tests for immigrants, and to alleged illegal voting by immigrants. Previous studies found that the fairness frame is more endorsed by the liberal than with the conservative (Graham, Haidt, \& Nosek, 2009). The dominance of conservative outlets in the study sample might contribute to the unpopularity of this frame. Third, the moral frame of care may produce contradictory behavioral effects: the audience dislikes the frame but tends to share it more. This frame set emphasizes the protection of individuals from harm and other forms of injustice (Graham et al., 2009). The studied content associated with this value tends to include words semantically and contextually similar to safe, peace, empathy, compassion, and others. The articles most representative of this moral frame are those discussing the protection of LGBTQ migrants and aging migrants (by liberal outlets), and Pope Francis's call to help poor immigrants (a position lauded by liberal outlets but viewed skeptically by conservative outlets). It is possible that the frame is associated with highly controversial issues that lead to negative sentiments toward the content. But the controversial content might be preferred in terms of sharing in order to provoke and to invoke moral outrage among the audience's like-minded peers.

The implication of our findings concerning moral frames is two-folded. First, it suggests that in deciding whether or not to share news articles, audiences likely pay attention to both the source and characteristics of actual content. The patterns concerning the appeals of specific moral frames seem to imply that audiences do look for ideological congruence (Metzger et al., 
2010; Meyer et al., 2010). This finding leads us to argue that in order to understand disinformation and partisanship on social media, we need to go beyond examining problematic platforms and practices. The current literature is constrained by its focus on state-sponsored information campaigns and fringe groups' systematic manipulation of customization algorithms for public attention (Marwick \& Lewis, 2017). While those are important factors to consider and such malpractices should be held accountable, overlooking what really causes political divides misses an opportunity to truly bridge schisms in society. Instead of simply de-legitimizing certain viewpoints and practices, this study follows Haidt's (2012) calls for a deeper understanding of partisan divides through the lens of moral foundations and examine how such divides are manifested on the micro-level in term of news framing. Currently, our findings are promising, adding further evidence to previous studies that link framing to news sharing and also confirming the salience of moral foundations in the current scholarly debates on disinformation and partisanship. Second, given the shareworthiness of certain moral frames, it is worth asking the following question: what role does social media algorithms play in amplifying specific moral frames? Scholars have long suspected recommendation algorithms as the culprit in reinforcing political biases and divides (Marwick \& Lewis, 2017), despite more recent evidence claiming that the echo chamber effects and filter bubbles that may result from algorithms might be overrated (Bruns, 2019; Weeks, Ksiazek, \& Holbert, 2016). It remains unclear to what extent moral frames are amplified or limited by algorithms digital platforms employ.

In view of the findings concerning how news content is related to audience engagement, we are aware of the fact that many audiences, when consuming news online, may not read beyond headlines and the first paragraph (Gabielkov et al., 2016). In the post-hoc analysis, we applied the same model to news titles and short descriptions of the news articles shared in 
Facebook posts. We identified several rather similar patterns: Titles and short descriptions that emphasize the support of authority/respect were significantly shared and liked more, whereas those focusing on the fairness/reciprocity frame were shared less. Likewise, content emphasizing the moral frame of care also received fewer likes. These findings suggest a high degree of consistency in moral frames used in the actual news content and in the Facebook posts that promote the news content. It may also suggest that the same audience engagement dynamics exist even for those skimming through the content.

\section{Limitations and future directions}

Readers are reminded of the following limitations of the study. First, the study does not provide a causal explanation, although we can speculate on multiple explanations of the findings based on the reviewed theoretical angles (i.e., news values, information processing, and media trust). Second, the study focuses solely on the coverage of immigration. While such a topical focus can effectively control for variations in topical selection that exist among outlets, it may skew some findings towards a particular political group because of the group's ability to sensationalize the issue through attention-grabbing headlines and content (e.g., linking immigration to crime vs. linking immigration to humanitarian crises). Third, outlets of different ideological camps may focus on different events related to immigration (e.g., crime, social welfare, the debate over multiculturalism, national security, etc.), which could result in different framing. Fourth, the creation of the list of hyper-partisan outlets itself is debatable. Conservatives and liberals have all accused the other side of spreading fake news. The list may include outlets that strive to do real journalism as well as "clickbait" sites whose mission is maximizing audience attention without attending to basic journalistic standards. Lumping them together may overlook important variations within the hyper-partisan media ecosystem. Fifth, the study assumes that when users 
share a news article, the users explicitly endorse the content. Nevertheless, it is possible that news sharing is for expressing disagreement. Due to the lack of data, our interpretations of audience motivations are limited. Sixth, the expired URLs may link to erroneous or controversial news content, which media outlets later deleted. This represents a lost opportunity to study the truly problematic side of hyper-partisan journalism. To expand the current research line, future studies can use a quasi-experiment on Facebook to test the effect of the four factors on news sharing. Future studies should also include a broader selection of issues and news events. Moreover, future studies need to consider adding additional news value variables to the model to account for the social construction of controversy, deviance, and stigma. 


\section{References}

Bakir, V., \& Mcstay, A. (2017). Digital Journalism Fake News and The Economy of Emotions, Digital Journalism, 6(2), 154-175.

Bennett, W. L., \& Livingston, S. (2018). The disinformation order: Disruptive communication and the decline of democratic institutions. European Journal of Communication, 33(2), 122-139.

Benkler, Y., Faris, R., \& Roberts, H. (2018). Network propaganda: Manipulation, disinformation, and radicalization in American politics. New York, NY: Oxford University Press.

Berger, J. (2011). Arousal increases social transmission of information. Psychological Science, 22, 891-893. Retrieved from http://doi.org/10.1177/0956797611413294

Brady, W. J., Wills, J. A., Jost, J. T., Tucker, J. A., \& Van Bavela, J. J. (2017). Emotion shapes the diffusion of moralized content in social networks. Proceedings of the National Academy of Sciences of the United States of America, 114, 7313-7318.

Chaiken, S., Liberman, A., \& Eagly, A. H. (1989). Heuristic and Systematic Information Processing within and beyond the Persuasion Context. In J. S. Uleman, \& J. A. Bargh (Eds.), Unintended Thought (pp. 212-252). New York, NY: Guilford.

Chadha, K., \& Koliska, M. (2015). Newsrooms and Transparency in the Digital Age. Journalism Practice, 9(2), 215-229.

Choma, B. L., \& Hanoch, Y. (2017). Cognitive ability and authoritarianism: Understanding support for Trump and Clinton. Personality and Individual Differences, 106, 287-291.

Chung, J. E. (2017). Retweeting in health promotion: Analysis of tweets about Breast Cancer Awareness Month. Computers in Human Behavior, 74, 112-119. 
Choi, W., \& Stvilia, B. (2015). Web credibility assessment: Conceptualization, operationalization, variability, and models. Journal of the Association for Information Science and Technology, 66(12), 2399-2414.

Coddington, M. (2014). Normalizing the hyperlink: How bloggers, professional journalists, and institutions shape linking values. Digital Journalism, 2(2), 140-155.

Curry, A., \& Stroud, N. J. (2017). Trust in Online News, 1-23. Retrieved from https://mediaengagement.org/wp-content/uploads/2017/12/CME-Trust-in-OnlineNews.pdf

De Vreese, C. H. (2012). New avenues for framing research. American Behavioral Scientist, $56(3), 365-375$

dos Santos Jr, M. A., Lycarião, D., \& de Aquino, J. A. (2019). The virtuous cycle of news sharing on Facebook: Effects of platform affordances and journalistic routines on news sharing. New Media \& Society, 21(2), 398-418.

Eilders, C. (2006). News factors and news decisions. Theoretical and methodological advances in Germany. Communications, 31, 5-24.

Entman, R. M. (1993). Framing: Toward clarification of a fractured paradigm. Journal of Communication, 43(4), 51-58.

Faris, R., Roberts, H., Etling, B., Bourassa, N., Zuckerman, E., \& Benkler, Y. (2017). Partisanship, propaganda, and disinformation: Online media and the 2016 US presidential election. Retrieved from https://cyber.harvard.edu/publications/2017/08/mediacloud

Figenschou, T. U., \& Ihlebæk, K. A. (2019). Challenging journalistic authority: Media criticism in far-right alternative media. Journalism Studies, 20(9), 1221-1237. 
Fisher, C. (2016). The trouble with 'trust' in news media. Communication Research and Practice, 2(4), 451-465.

Fletcher, R., \& Park, S. (2017). The impact of trust in the news media on online news consumption and participation. Digital Journalism, 5(10), 1281-1299.

Gabielkov M, Ramachandran A, Chaintreau A, et al. (2016) Social clicks: What and who gets read on Twitter? In: Proceedings of the ACM 2016 sigmetrics international conference on measurement and modeling of computer science, pp. 179-192. Available at: https://hal.inria. fr/hal-01281190/document

García-Perdomo, V., Salaverría, R., Kilgo, D. K., \& Harlow, S. (2018). To share or not to share: The influence of news values and topics on popular social media content in the United States, Brazil, and Argentina. Journalism Studies, 19(8), 1180-1201.

Garten, J., Hoover, J., Johnson, K. M., Boghrati, R., Iskiwitch, C., \& Dehghani, M. (2018). Dictionaries and distributions: Combining expert knowledge and large scale textual data content analysis. Behavior Research Methods, 50(1), 344-361.

Garten, J., Boghrati, R., Hoover, J., Johnson, K. M., \& Dehghani, M. (2016, July). Morality between the lines: Detecting moral sentiment in text. In Proceedings of IJCAI 2016 workshop on Computational Modeling of Attitudes.

Haidt, J. (2012). The Righteous Mind: Why Good People are Divided by Politics and Religion. London: Allen Lane.

Haidt, J., \& Graham, J. (2007). When morality opposes justice: Conservatives have moral intuitions that liberals may not recognize. Social Justice Research, 20(1), 98-116.

Graham, J., Haidt, J., \& Nosek, B. A. (2009). Liberals and conservatives rely on different sets of moral foundations. Journal of Personality and Social Psychology, 96(5), 1029-1046. 
Harber, K. D., \& Cohen, D. J. (2005). The emotional broadcaster theory of social sharing. Journal of Language and Social Psychology, 24, 382-400.

Holt, K., Ustad Figenschou, T., \& Frischlich, L. (2019). Key Dimensions of Alternative News Media, Digital Journalism, Advanced online publication, doi: $10.1080 / 21670811.2019 .1625715$.

Hoover, J., Johnson, K., Boghrati, R., Graham, J., \& Dehghani, M. (2018). Moral framing and charitable donation: Integrating exploratory social media analyses and confirmatory experimentation. Collabra: Psychology, 4(1), 9.

Jahng, M. R., \& Littau, J. (2016). Interacting is believing: Interactivity, social Cue, and perceptions of journalistic credibility on Twitter. Journalism \& Mass Communication Quarterly, 93(1), 38-58.

Jutel, O. (2018). Donald Trump, American populism and affective media. In C. de la Torre (Ed.), Routledge Handbook of Global Populism (pp. 249-262). New York, NY: Routledge.

Kalogeropoulos, A., Negredo, S., Picone, I., \& Nielsen, R. K. (2017). Who shares and comments on news?: A cross-national comparative analysis of online and social media participation. Social Media+ Society, 3(4), 2056305117735754.

Khuntia, J., Sun, H., \& Yim, D. (2016). Sharing news through social networks. JMM International Journal on Media Management, 18(1), 59-74.

Kilgo, D. K., Lough, K., \& Riedl, M. J. (2017). Emotional appeals and news values as factors of shareworthiness in Ice Bucket Challenge coverage. Digital Journalism, Advanced online publication. doi: 10.1080/21670811.2017.1387501

Kim, H. S. (2015). Attracting views and going viral: How message features and news-sharing channels affect health news diffusion. Journal of Communication, 65(3), 512-534. 
Knight Foundation \& Gallup. (2018). Indicators of news media trust. Knight Foundation. Retrieved from https://www.knightfoundation.org/reports/indicators-of-newsmedia-trust

Kümpel, A. S., Karnowski, V., \& Keyling, T. (2015). News sharing in social media: A review of current research on news sharing users, content, and networks. Social Media+ Society, $1(2), 1-14$.

Landreville, K. D., \& Niles, C. (2019). “And That'sa Fact!”: The Roles of Political Ideology, PSRs, and Perceived Source Credibility in Estimating Factual Content in Partisan News. Journal of Broadcasting \& Electronic Media, 63(2), 177-194.

Lee, J. (2015). The double-edged sword: The effects of journalists' social media activities on audience perceptions of journalists and their news products. Journal of ComputerMediated Communication, 20(3), 312-329.

Lee, C. S., \& Ma, L. (2012). News sharing in social media: The effect of gratifications and prior experience. Computers in Human Behavior, 28(2), 331-339.

Lischka, J. A. (2019). A badge of honor? How The New York Times discredits president Trump's fake news accusations. Journalism Studies, 20(2), 287-304.

Lind, M. (2020). The new class War: Saving democracy from the managerial elite. Portfolio.

Liu, Y., Rui, J. R., \& Cui, X. (2017). Are people willing to share their political opinions on Facebook? Exploring roles of self-presentational concern in spiral of silence. Computers in Human Behavior, 76, 294-302.

Liu, J., Li, C., Ji, Y. G., North, M., \& Yang, F. (2017). Like it or not: The Fortune 500's Facebook strategies to generate users' electronic word-of-mouth. Computers in Human Behavior, 73, 605-613. 
Lowry, D. T. (2008). Network TV news framing of good vs. bad economic news under Democrat and Republican presidents: A lexical analysis of political bias. Journalism \& Mass Communication Quarterly, 85(3), 483-498.

Marwick, A., \& Lewis, R. (2017). Media manipulation and disinformation online. Retrieved from https://datasociety.net/output/media-manipulation-and-disinfo-online/

Metzger, M. J., Hartsell, E. H., \& Flanagin, A. J. (2015). Cognitive dissonance or credibility? A comparison of two theoretical explanations for selective exposure to partisan news. Communication Research, Advance online publication. doi: 10.1177/0093650215613136.

Meyer, H. K., Marchionni, D., \& Thorson, E. (2010). The journalist behind the news: Credibility of straight, collaborative, opinionated, and blogged "news." American Behavioral Scientist, 54(2), 100-119.

Mourão, R. R., \& Robertson, C. T. (2019). Journalism Studies Fake News as Discursive Integration: An Analysis of Sites That Publish False, Misleading, Hyperpartisan and Sensational Information. Journalism Studies, doi:10.1080/1461670X.2019.1566871

Mor, N., \& Reich, Z. (2018). From "Trust Me" to "Show Me" Journalism: Can DocumentCloud help to restore the deteriorating credibility of news?. Journalism Practice, 12(9), 10911108.

Nielsen, R., Newman, N.., Fletcher, R., \& Kalogeropoulos, A. (2019). Reuters Institute Digital News Report 2019. Retrieved from https://reutersinstitute.politics.ox.ac.uk/sites/default/files/201906/DNR_2019_FINAL_0.pdf

Papacharissi, Z. (2015). Affective publics: Sentiment, technology, and politics. New York: Oxford University Press. 
Pennebaker, J. W., Boyd, R. L., Jordan, K., \& Blackburn, K. (2015). The development and psychometric properties of LIWC2015.

Perrin, A., \& Anderson, M. (2019). Share of U.S. adults using social media, including Facebook, is mostly unchanged since 2018. Pew Research Center, Retrieved from https://www.pewresearch.org/fact-tank/2019/04/10/share-of-u-s-adults-using-socialmedia-including-facebook-is-mostly-unchanged-since-2018/

Peters, C. (2011). Emotion aside or emotional side? Crafting an 'experience of involvement' in the news. Journalism, 12(3), 297-316.

Pincus, H., Wojcieszak, M., \& Boomgarden, H. (2017). Do multimedia matter? Cognitive and affective effects of embedded multimedia journalism. Journalism \& Mass Communication Quarterly, 94(3), 747-771.

Rowley, J., \& Johnson, F. (2013). Understanding trust formation in digital information sources: The case of Wikipedia. Journal of Information Science, 39(4), 494-508.

Ryan, C., Carragee, K. M., \& Meinhofer, W. (2010). Theory into practice: Framing, the news media, and collective action. Journal of Broadcasting \& Electronic Media, 45(1), 175182.

Semetko, H. A., \& Valkenburg, P. M. (2000). Framing European politics: A content analysis of press and television news. Journal of Communication, 50(2), 93-109.

Silverman, C. (2018, November 16). This analysis shows how viral fake election news stories outperformed real news on Facebook. BuzzFeed News, Retrieved from https://www.buzzfeednews.com/article/craigsilverman/viral-fake-election-newsoutperformed-real-news-on-facebook 
Stieglitz, S., \& Dang-Xuan, L. (2012, January). Political communication and influence through microblogging--An empirical analysis of sentiment in Twitter messages and retweet behavior. In System Science (HICSS), 2012 45th Hawaii International Conference on (pp. 3500-3509). IEEE.

Sundar, S. S. (2008). The MAIN model: A heuristic approach to understanding technology effects on credibility. Digital media, youth, and credibility, 73100.

Tandoc, E. C., Lim, W., \& Ling, R. (2017). Defining "fake news": A typology of scholarly definitions. Digital Journalism, 6(2), 137-153.

Trilling, D., Tolochko, P., \& Burscher, B. (2017). From newsworthiness to shareworthiness: How to predict news sharing based on article characteristics. Journalism \& Mass Communication Quarterly, 94(1), 38-60.

Tucker, J., Guess, A., Barberá, P., Vaccari, C., Siegel, A., Sanovich, S., ... \& Nyhan, B. (2018). Social media, political polarization, and political disinformation: A review of the scientific literature. Retrieved from https://hewlett.org/library/social-media-politicalpolarization-political-disinformation-review-scientific-literature/

Valenzuela, S., Piña, M., \& Ramírez, J. (2017). Behavioral effects of framing on social media users: How conflict, economic, human interest, and morality frames drive news sharing. Journal of Communication, 67(5), 803-826.

Veltri, G. A., \& Atanasova, D. (2017). Climate change on Twitter: Content, media ecology and information sharing behaviour. Public Understanding of Science, 26(6), 721-737.

Walther, J. B. (1993). Impression development in computer-mediated interaction. Western Journal of Communication, 57(4), 381-398.

Wang, M. Y., Hmielowski, J. D., Hutchens, M. J., \& Beam, M. A. (2017). Extending the spiral 
of silence: partisan media, perceived support, and sharing opinions online. Journal of Information Technology \& Politics, 14(3), 248-262. 